\title{
Neonatos críticos recebem a terapia nutricional parenteral em volume conforme suas necessidades prescritas? Estudo clínico observacional
}

\author{
Critical neonates receive parenteral nutritional therapy in volume according to their prescribed \\ needs? Observational clinical study
}

DOI: $10.37111 /$ braspenj.2020354006

Daniela Alencar Moreira

Neyla Edelwais Silva ${ }^{2}$

Cervantes Caporossi ${ }^{3}$

\section{Unitermos:}

Nutrição Parenteral. Recém-nascido. Farmacoeconomia. Unidades de Terapia Intensiva Neonatal. Serviço de Farmácia Hospitalar.

\section{Keywords:}

Parenteral Nutrition. Infant, Newborn. Economics, Pharmaceutical. Intensive Care Units, Neonatal. Pharmacy Service, Hospital.

\section{Endereço para correspondência:}

Daniela Alencar Moreira

Hospital Universitário Júlio Muller - Setor de Farmácia Hospitalar

Rua Luís Philippe Pereira Leite, s/n - Alvorada Cuiabá, MT, Brasil - CEP 78048-902

E-mail: dani.alencar@hotmail.com;

daniela.alencar@ebserh.gov.br

\section{Submissão}

9 de novembro de 2020

Aceito para publicação

11 de dezembro de 2020
RESUMO

Introdução: $O$ monitoramento da terapia de nutrição parenteral (TNP) em neonatos críticos é essencial para o desfecho clínico, sendo fundamental quantificar a TNP infundida comparada com a quantidade prescrita e preparada. O objetivo deste estudo é contabilizar as perdas monetárias devido ao descarte de TNP não administrada. Método: Os neonatos foram estratificados em dois grupos. Grupo $A(n=6)$ : prematuros de baixo peso; Grupo B $(n=6)$ : prematuros de muito baixo peso e extremo baixo peso $<1.000 \mathrm{~g}$, sendo acompanhados por 167 dias de TNP, comparados pelas diferenças percentuais dos volumes, calorias e média das perdas monetárias dos volumes desprezados. Resultados: A diferença percentual entre volume prescrito e preparado para o grupo A foi de $42,49 \pm 18,27 \%$ e para o grupo $B$ foi de $86,82 \pm 63,52 \%(p=0,003)$. Entre volume preparado e infundido, a diferença foi de $63,15 \pm 15,11 \%$ para o grupo $A$ e $47,98 \pm 17,25 \%$ para $\circ$ grupo $B(p=0,003)$. Entre calorias prescritas e infundidas, a diferença foi de $22,61 \pm 8,69 \%$ para o grupo $A$ e $13,92 \pm 3,45 \%$ para o grupo $B(p=0,04)$. As médias de perdas monetárias dos volumes desprezados foram $R \$ 992,68$ e $R \$ 1.296,81$ ( $p=0,0129)$, respectivamente. Conclusão: A TNP infundida foi inferior à prescrita, resultando em menor aporte calórico para os neonatos e perdas financeiras significativas associadas ao descarte de TNP.

\section{ABSTRACT}

Introduction: Monitoring of parenteral nutrition therapy (NPT) in critical neonates is essential for clinical outcome, and it is indispensable to quantify the infused NPT compared to the prescribed and prepared amount. The purpose of this study is to account for monetary losses due to unmanaged NPT disposal. Methods: Neonates were stratified into two groups. Group A (n $=6)$ : low birth weight preterm infants; Group $B(n=6)$ : preterm infants of very low weight and extreme low weight $<1,000 \mathrm{~g}$, followed by 167 days of NPT, compared to the percentage differences in volumes, calories and average monetary losses of the despised volumes. Results: The percentage difference between volume prescribed and prepared for group $A$ was $42.49 \pm$ $18.27 \%$ and for group $B$ it was $86.82 \pm 63.52 \%(p=0.003)$. Between volume prepared and infused, the difference was $63.15 \pm 15.11 \%$ for group $A$ and $47.98 \pm 17.25 \%$ for group $B$ ( $p$ $=0.003)$. Among prescribed and infused calories, the difference was $22.61 \pm 8.69 \%$ for group $A$ and $13.92 \pm 3.45 \%$ for group $B(p=0.04)$. The average monetary losses of the despised volumes were R\$992.68 and R\$1,296.81 ( $p=0.0129)$, respectively. Conclusion: Infused NPT was lower than prescribed, resulting in lower caloric intake for neonates and significant financial losses associated with NPT disposal.

1. Mestre, Especialista em Manipulação Alopática Magistral, Farmacêutica da equipe multidisciplinar de terapia nutricional do Hospital Universitário Júlio Muller/ Universidade Federal de Mato Grosso, Programa de Mestrado Profissional, Cuiabá, MT, Brasil.

2. Mestre, Especialista em Nutrição Clínica, Parenteral e Enteral, nutricionista da equipe multidisciplinar de terapia nutricional do Hospital Universitário Júlio Muller/ Universidade Federal de Mato Grosso, Programa de Mestrado Profissional, Cuiabá, MT, Brasil.

3. Doutor, Universidade Federal de Mato Grosso, Faculdade de Medicina, Programa de Pós-graduação em Ciências Aplicadas à Gestão Hospitalar (PPGCAAH), Hospital Santa Rosa, Cuiabá, MT, Brasil. 


\section{INTRODUÇÃO}

A terapia nutricional parenteral (TNP) é essencial para suprir as necessidades calóricas, repor elementos nutricionais e modular as respostas inflamatórias de pacientes críticos hospitalizados. Em neonatos prematuros ou gravemente doentes, exerce papel fundamental nas funções do sistema imunológico, além de promover o crescimento e desenvolvimento das estruturas imaturas'

A terapia nutricional deve ser iniciada o mais precocemente possível (24 a 48 horas), em recém-nascidos admitidos em Unidade de Terapia Intensiva (UTI) neonatal. Geralmente é observada em três fases distintas: fase de nutrição parenteral exclusiva, fase de transição, quando a nutrição parenteral está associada à nutrição enteral, e a fase de nutrição enteral isolada, quando a alimentação do neonato está bem estabelecida de forma enteral, preferencialmente com o leite materno ${ }^{2-5}$.

Para uma TNP segura em pacientes críticos, vários processos essenciais devem ser avaliados. $\bigcirc$ cálculo exato das necessidades do paciente, a composição adequada da TNP e o rígido controle da administração devem ser acompanhados, pois, diferenças podem colaborar para possíveis complicações relacionadas à TNP, inclusive estabelecer uma desnutrição intra-hospitalar ${ }^{4,6-9}$.

Segundo Cotogni ${ }^{6}$, falhas no processo de administração da TNP estão relacionadas, principalmente, às taxas de infusão discordantes da prescrita. Alterações metabólicas graves podem ocorrer, hiperglicemia, sobrecarga de lipídeos ou, eventualmente, redução do volume infundido, com déficit nutricional, manutenção de um balanço de nitrogênio negativado e, consequentemente, um agravo nutricional ${ }^{10}$.

Apesar da administração da TNP (associada ou não à enteral) ser tecnicamente controlada no ambiente hospitalar, a literatura descreve que, de forma significativa, a quantidade administrada é, em grande percentual de pacientes, inferior ao volume determinado na prescrição e, consequentemente, também para as calóricas calculadas como necessárias aos pacientes $^{9-11}$.

A solução de TNP, prescrita e preparada de forma individualizada para cada paciente, é uma formulação extemporânea, ou seja, possui tempo limitado de conservação. Quando preparada e não administrada no paciente, deve ser descartada no intervalo de 24 horas $^{12}$. Isto pode impactar negativamente para a Instituição e, assim, este indicador fármaco-econômico deve ser mensurado e avaliado, objetivando a redução de custos e minimização dos prejuízos econômicos para a unidade hospitalar.

Dock-Nascimento et al. ${ }^{9}$, avaliando pacientes adultos, relatam que aproximadamente $30 \%$ do que é prescrito da TNP não é ofertado, ou seja, ocorrem perdas quantitativas do material preparado, fato que implica em uma oferta parcial das necessidades. Pressupõe-se que esta realidade deve ser estendida para todos os pacientes críticos, independente da sua idade e unidade onde o serviço é prestado. Assim sendo, faz-se necessário um estudo orientado para avaliação dos volumes $(\mathrm{mL}$ ) e das calorias ( $\mathrm{kcal}$ ) da TNP que são prescritos, preparados na farmácia e infundidos no paciente neonatos críticos. Concomitantemente, este estudo busca contabilizar possíveis perdas monetárias para a Instituição devido ao descarte de TNP preparada e não administrada.

\section{MÉTODO}

Estudo prospectivo, observacional e analítico, realizado no Hospital Universitário Júlio Müller (HUJM), submetido e aprovado pelo Comitê de Ética em Pesquisa, com CAAE $n^{\circ}$ 59580316.8.0000.5541. Este hospital atende a pacientes neonatos prematuros críticos, geralmente com infecção grave. Aproximadamente 40\% dos pacientes internados na UTI Neonatal recebem TNP. A farmácia do HUJM é responsável pela produção da solução de nutrição parenteral, com preparo médio de 100 bolsas de TNP para neonatos prematuros/mês, número este que corresponde a $59 \%$ do total produzido no serviço.

Os critérios de elegibilidade para participar desta pesquisa foram: pacientes neonatos prematuros, com idade gestacional de até 36 semanas e 6 dias, com peso máximo ao nascer de $2.499 \mathrm{~g}$, internados na UTI Neonatal, em TNP exclusiva ou associada de outra terapia nutricional concomitante, seja enteral ou amamentação com leite materno ou artificial. Foram excluídos os RNs transferidos para o HUJM.

A estratificação dos neonatos foi realizada conforme orientação da Organização Mundial de Saúde (OMS), sendo classificados quanto à prematuridade, respectivamente, em: pré-termos extremos (idade gestacional - IG < 30 semanas); muito prematuros (IG de 30 a 33 semanas e 6 dias); pré-termos tardios (IG de 34 a 36 semanas e 6 dias); e com relação ao peso de nascimento em: extremo baixo peso (recém-nascidos $<1.000 \mathrm{~g}$ ); muito baixo peso (recém-nascidos com peso entre 1.000 a $1.499 \mathrm{~g}$ ); baixo peso (recém-nascidos com peso entre 1.500 a $2.499 \mathrm{~g})^{13}$.

Os pacientes foram diariamente avaliados em relação às seguintes variáveis: volume prescrito, volume preparado, volume infundido, diferença entre volume prescrito e infundido, diferença entre volume preparado e infundido, perdas monetárias dos volumes desprezados, calorias prescritas, infundidas e diferença entre elas.

O estudo foi elaborado em duas etapas. Inicialmente, a diferença entre as variáveis (média $\pm \mathrm{DP}$ ) foi avaliada usando dados coletados em cada dia de administração da TNP, ou seja, uma somatória de dias, independentemente do paciente e do número de dias de sua participação. Posteriormente, foi realizada uma avaliação estratificada por grupo de pacientes. 
A variação de interesse no estrato foi um número de dias consecutivos de uso de TNP em que fosse incluída a maior camada de representantes (tempo uniforme de TNP - 6 dias).

Após a definição da amostra, os RNs elencados foram subdivididos em dois grupos, conforme o peso de nascimento: grupo $A(n=6)$ : prematuros de baixo peso (1.500 a $2.499 \mathrm{~g})$ e grupo $B(\mathrm{n}=6)$ : prematuros de muito baixo peso ( 1.000 a $1.499 \mathrm{~g})$ e extremo baixo peso $(<1.000 \mathrm{~g})$.

Os grupos foram comparados e avaliados estatisticamente quanto às seguintes variáveis: diferença percentual entre volume prescrito e preparado, prescrito e infundido, preparado e infundido, diferença de calorias prescritas e infundidas nos seis dias iniciais de terapia, diferença percentual de peso corpóreo do início da terapia ao $6^{\circ}$ dia de terapia e média diária das perdas monetárias nos seis dias de TNP. Nesta etapa, três pacientes foram excluídos do estudo por não completarem os seis dias iniciais consecutivos de terapia.

Foi avaliada a média das perdas diárias representada pelo volume $(\mathrm{mL})$ preparado e o volume não administrado (desprezado) e, posteriormente, calculado o valor $(R \$)$ da fração eliminada. Utilizou-se o valor de $R \$ 5,12$ (cinco reais e doze centavos) para cada $\mathrm{mL}$ da solução de TNP, conforme cotação realizada em uma empresa privada local. Para determinar este valor, considera-se todo o múltiplo aparato que permite a complexa administração da TNP, envolvendo os macros e micronutrientes da sua composição, como também todos os equipamentos e componentes necessários para uma segura e contínua infusão da solução.

Na farmácia do HUJM, são preparadas diariamente as soluções de TNP, em única etapa para 24 horas. O protocolo de manipulações de TNP para pacientes neonatos estabelece, em adição à solução prescrita, um volume de $55 \mathrm{~mL}$ para cada formulação. $O$ protocolo vigente justifica este rotineiro avolumar para cumprir com as finalidades elencadas a seguir: $25 \mathrm{~mL}$ para preenchimento do equipo de bomba de infusão; $10 \mathrm{~mL}$ para testes de controle de qualidade (amostras de prova e contraprova); e os demais $20 \mathrm{~mL}$ para possíveis perdas de solução durante o processo. Sendo assim, após a manipulação da TNP, são aspirados $10 \mathrm{~mL}$ de cada bolsa, destinados aos testes de controle de qualidade das soluções ${ }^{12}$.

$\mathrm{Na}$ estatística descritiva, foram calculadas as médias, medianas, mínimo, máximo e desvio-padrão de todas as variáveis. Para o cálculo das diferenças entre os volumes prescritos, preparados e infundidos, bem como das diferenças entre calorias prescritas e infundidas, utilizou-se o teste $t$ de Student para dados pareados. A homogeneidade das variâncias foi testada pelo teste de Levene, enquanto para o teste de normalidade utilizou-se o método de Kolmogorov-Smirnov.

Após a estratificação dos grupos, como todas as distribuições satisfizeram os pressupostos de homocedasticidade e normalidade, as diferenças das médias foram avaliadas por meio do teste ANOVA fator duplo com repetição e teste post hoc através do método de Tukey. Para o cálculo das perdas monetárias dos volumes desprezados, percentagem da diferença de calorias prescritas e infundidas e percentagem da diferença de peso do início ao $6^{\circ}$ dia de terapia, utilizou-se o teste + de Student para dados não pareados.

\section{RESULTADOS}

\section{Ełapa 1 - Análise Considerando Dias de TNP}

Participaram deste estudo um total de 15 pacientes, que foram acompanhados por 167 dias de TNP, no período de outubro a dezembro de 2016 . O peso médio dos neonatos ao nascer foi de 1.3476 gramas ( \pm 0,559 gramas). A Tabela 1 apresenta os dados demográficos e características clínicas dos participantes.

\begin{tabular}{|c|c|c|}
\hline Características & $\begin{array}{c}N^{0} \text { participantes } \\
(n=15)\end{array}$ & $(\%)$ \\
\hline \multicolumn{3}{|l|}{ Sexo } \\
\hline Feminino & 8 & 53,33 \\
\hline Masculino & 7 & 46,67 \\
\hline \multicolumn{3}{|c|}{ Conforme a prematuridade } \\
\hline Pré-termos extremos & 8 & 53,33 \\
\hline Muito prematuros & 4 & 26,67 \\
\hline Pré-termos tardios & 3 & 20,00 \\
\hline \multicolumn{3}{|c|}{ Conforme o peso de nascimento } \\
\hline Extremo baixo peso & 5 & 33,33 \\
\hline Muito baixo peso & 3 & 20,00 \\
\hline Baixo peso & 7 & 46,67 \\
\hline \multicolumn{3}{|l|}{ Tipo de parto } \\
\hline Vaginal & 2 & 13,33 \\
\hline Cesáreo & 13 & 86,67 \\
\hline \multicolumn{3}{|l|}{ Surfactante pulmonar } \\
\hline $\operatorname{Sim}$ & 12 & 80,00 \\
\hline Não & 3 & 20,00 \\
\hline \multicolumn{3}{|l|}{ Tipo de acesso venoso } \\
\hline Cateter umbilical & 8 & 53,33 \\
\hline Cateter de PICC & 6 & 40 \\
\hline Periférico & 1 & 6,67 \\
\hline \multicolumn{3}{|c|}{$\begin{array}{l}\text { Dieta enteral, aleitamento } \\
\text { materno ou artificial }\end{array}$} \\
\hline Sim & 12 & 80,00 \\
\hline Não & 3 & 20,00 \\
\hline \multicolumn{3}{|l|}{ Desfecho clínico } \\
\hline Alta melhorada & 12 & 80,00 \\
\hline Óbito & 3 & 20,00 \\
\hline
\end{tabular}


A Tabela 2 apresenta os dados coletados quanto aos volumes prescritos, preparados e infundidos, as diferenças médias entre volume prescrito e infundido e volume preparado e infundido, bem como as perdas monetárias conforme volumes desprezados de TNP preparada e não administrada.

Observam-se volumes significativos de TNP desprezados, os quais foram preparados, porém não foram administrados nos pacientes. Este fato pode ser confirmado na variável diferença entre o volume preparado e infundido, com consequentes perdas monetárias importantes (Tabela 2).

A Tabela 3 apresenta as médias de calorias prescritas, calorias infundidas e a diferença entre calorias prescritas e infundidas.

\begin{tabular}{lccccc}
\hline \multicolumn{5}{ll}{ Tabela 2 - Volumes prescritos, preparados e infundidos (média \pm DP) } & avaliação das perdas monetárias (volumes desprezados) nos 167 dias de TNP. \\
\hline Variável & Média & Mediana & Mínimo & Máximo & Valor-p \\
\hline Volume prescrito* & $111,9 \pm 56,1$ & 106,8 & 19,0 & 260,4 & \\
Volume preparado* & $166,2 \pm 56,8$ & 160,5 & 74,0 & 315,4 & 228,2 \\
Volume infundido* & $87,1 \pm 49,3$ & 83,4 & - & 184,4 & 0,0001 \\
$\begin{array}{l}\text { Diferença entre volume prescrito e } \\
\text { infundido* }\end{array}$ & $24,3 \pm 18,9$ & 18,5 & 0,2 & & $<0,001$ \\
$\begin{array}{l}\text { Diferença entre volume preparado e } \\
\text { infundido* }\end{array}$ & $79,1 \pm 17,9$ & 74,3 & $-12,3$ & 210,6 & \\
$\begin{array}{l}\text { Perdas monetárias dos volumes } \\
\text { desprezados }\end{array}$ & $\mathrm{R} \$ 203,1 \pm 56,6^{* \star}$ & $\mathrm{R} \$ 198,9$ & $\mathrm{R} \$ 26,9$ & $\mathrm{R} \$ 953,2$ & \\
\hline
\end{tabular}

$\mathrm{TNP}=$ terapia nutricional parenteral; ${ }^{*}$ dados em mililitros; ${ }^{*}$ perdas em reais $(\mathrm{R} \$)$.

Tabela 3 - Diferença entre calorias (média \pm DP) prescritas e infundidas nos 167 dias de TNP.

\begin{tabular}{|c|c|c|c|c|c|}
\hline Variável & Média & Mediana & Mínimo & Máximo & Valor-p \\
\hline $\begin{array}{l}\text { Diferença entre calorias prescritas e infun- } \\
\text { didas }(\mathrm{kcal} / \mathrm{kg})\end{array}$ & $12,9 \pm 7,0$ & 9,4 & 2,0 & 83,1 & $<0,001$ \\
\hline
\end{tabular}

Quanto às perdas calóricas, verificam-se diferenças entre calorias prescritas e infundidas que, apesar de pequenas, são significantes $(p<0,001)$ e importantes para esta população de pacientes, em virtude de sua condição intrínseca relacionada ao seu baixo peso corpóreo ao nascer (Tabela 3).

\section{Etapa 2 - Análise Considerando Amostra de Pa- cientes com Seis Dias Completos de TNP}

Quando estudados os RN que completaram 6 dias de TNP e realizada a estratificação dos participantes em grupo $A$ (prematuros de baixo peso) e grupo $B$ (prematuros de muito baixo peso e extremo baixo peso), foi possível comparálos, com resultados expressos em percentagem, quanto às médias das diferenças de volumes: prescritos, preparados e infundidos (Figura 1 e Tabela 4).

A Tabela 4 apresenta as diferenças em percentagem dos volumes prescritos, preparados, infundidos do grupo $A$ e do grupo $B$, bem como a diferença de calorias prescritas e infundidas, a diferença de peso corpóreo de ambos os grupos do início ao sexto dia de TNP e as médias diárias das perdas monetárias dos volumes desprezados.

As variáveis relacionadas aos volumes e às calorias estudadas foram diferentes entre o grupo $A$ e o grupo $B(p<0,05)$. Observam-se perdas monetárias significativas entre os dois grupos $(p=0,0129)$, sendo apresentadas as médias diárias das perdas monetárias em reais $(R \$)$, no período de seis dias de TNP (Tabela 4). 


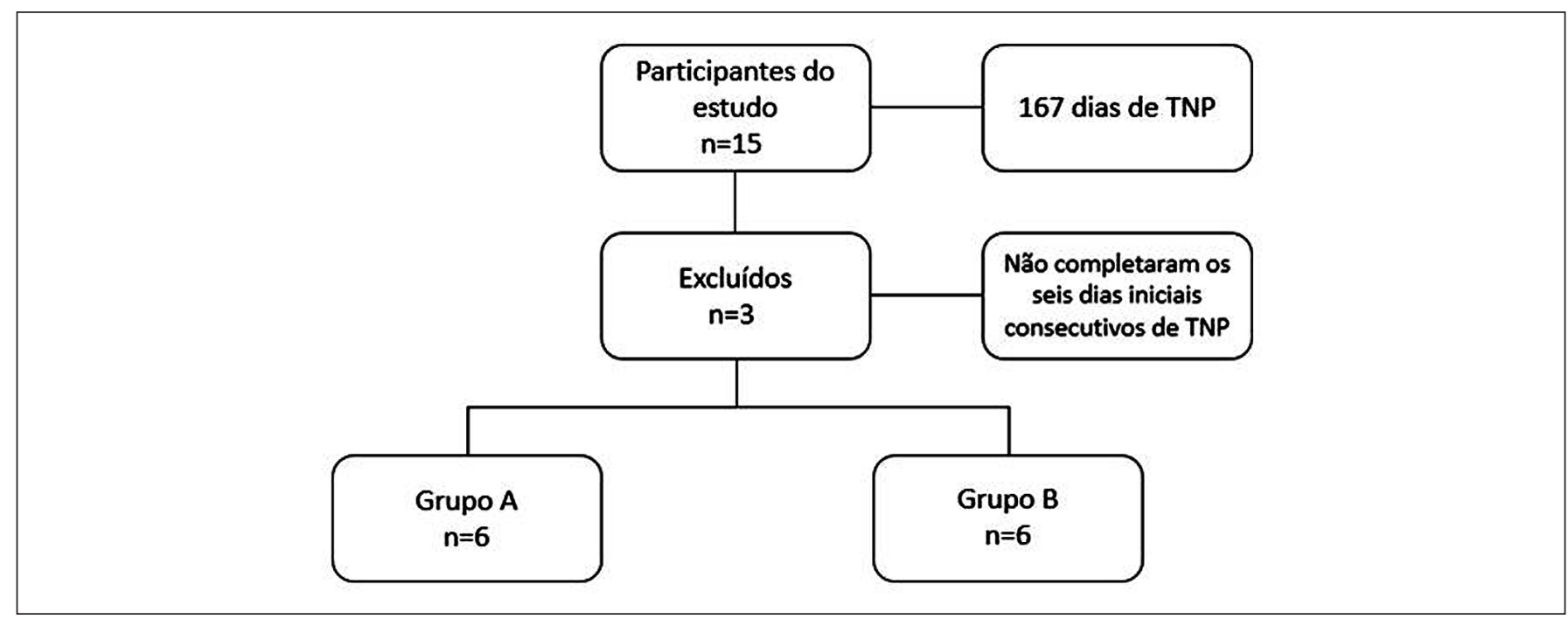

Figura 1 - Fluxograma do estudo estratificado com pacientes que completaram 6 dias consecutivos de TNP.

Tabela 4 - Diferença em percentagem (\%) de volumes prescritos, preparados, infundidos, calorias prescritas e infundidas, peso corpóreo em grupos de pacientes prematuros e avaliação das perdas monetárias (volumes desprezados) - 12 pacientes acompanhados por 6 dias de TNP.

\begin{tabular}{|c|c|c|}
\hline Variável & Média & Valor-p \\
\hline \multicolumn{3}{|c|}{ Diferença entre volume prescrito e preparado* } \\
\hline Grupo A & $42,49 \% \pm 18,27$ & 0,0003 \\
\hline Grupo B & $86,82 \% \pm 63,52$ & \\
\hline \multicolumn{3}{|c|}{ Diferença entre volume prescrito e infundido* } \\
\hline Grupo A & $88,72 \% \pm 19,91$ & 0,0732 \\
\hline Grupo B & $80,92 \% \pm 17,76$ & \\
\hline \multicolumn{3}{|c|}{ Diferença entre volume preparado e infundido* } \\
\hline Grupo A & $63,15 \% \pm 15,11$ & 0,0003 \\
\hline Grupo B & $47,98 \% \pm 17,25$ & \\
\hline \multicolumn{3}{|c|}{ Diferença de calorias prescritas e infundidas nos 6 dias iniciais de terapia* } \\
\hline Grupo A & $(-) 22,61 \% \pm 8,69$ & 0,04 \\
\hline Grupo B & $(-) 13,92 \% \pm 3,45$ & \\
\hline \multicolumn{3}{|c|}{ Diferença de peso do início da terapia e $6^{\circ}$ dia de terapia* } \\
\hline Grupo A & $(-) 3,98 \% \pm 6,57$ & 0,7928 \\
\hline Grupo B & $(-) 5,10 \% \pm 7,69$ & \\
\hline \multicolumn{3}{|c|}{ Perdas monetárias dos volumes desprezados } \\
\hline Grupo A & $\mathrm{R} \$ 992,68^{* *}$ & 0,0129 \\
\hline Grupo B & $\mathrm{R} \$ 1.296,81^{\star \star}$ & \\
\hline
\end{tabular}

\section{DISCUSSÃO}

Neste estudo, foi demonstrado que, no procedimento diário da TNP em neonatos críticos, os volumes infundidos são menores que os prescritos, resultando em provável prejuízo do aporte calórico para os pacientes. Do mesmo modo, perdas financeiras significativas são associadas ao descarte de TNP preparada e não administrada.

De acordo com o consenso Australiano ${ }^{14}$, de 2012, a necessidade calórica nutricional recomendada varia de 89 a $120 \mathrm{kcal} / \mathrm{kg} /$ dia em RNs prematuros, mesmo assumindo que as necessidades energéticas mínimas podem ser atendidas com 50 a $60 \mathrm{kcal} / \mathrm{kg} / \mathrm{dia}^{14}$. Um RN recebendo TNP necessita menos calorias que um RN alimentado por via enteral, porque não há energia perdida nas fezes, há menos termogênese, além de gasto energético com a sucção em casos de aleitamento materno. Da mesma forma, Pappoe et al. ${ }^{15}$ demonstraram, em um estudo clínico randomizado, que o aporte calórico energético precoce em bebês prematuros, 
com início de TNP de $49 \mathrm{kcal} / \mathrm{kg} / \mathrm{dia}$, aumentando para 75-80 kcal/kg/dia no segundo dia, resultaram significativamente em menos perda de peso pós-natal, e melhor ganho de peso na primeira semana de vida dos pacientes prematuros de muito baixo peso, associado a balanço nitrogenado positivo e tolerância à glicose ${ }^{15}$.

Neste estudo, foram identificadas a média das calorias prescritas em 50,6 $\pm 10,1 \mathrm{kcal} / \mathrm{kg}$ e a média das calorias infundidas de 37,7 $\pm 15 \mathrm{kcal} / \mathrm{kg}$ (Tabela 3), sendo constatadas perdas calóricas de 12,9 $\pm 7 \mathrm{kcal} / \mathrm{kg}$ (média das diferenças entre calorias prescritas e infundidas), $\operatorname{com} p<0,001$, confirmando que as necessidades calóricas mínimas diárias dos pacientes não foram totalmente atingidas. Esta menor infusão do volume de TNP e, consequentemente, menor ingesta de nutrientes, pode desencadear provável desnutrição intra-hospitalar, gerando perdas assistenciais.

Quando estratificados os participantes em dois grupos, grupos A $(n=6)$ e grupo $B(n=6)$, e avaliados pelos 6 primeiros dias de TNP, as diferenças de volumes e consequentes perdas calóricas ficam mais evidentes.

As perdas calóricas podem influenciar diretamente na perda de peso ou no não ganho de peso esperado nos participantes. Esta condição pode ser observada com a variável diferença de peso corpóreo no início e $6^{\circ}$ dia de terapia, resultando em perdas de -3,98\% e - $5,10 \%$, respectivamente, nos grupos A e B. Entretanto, deve-se ressaltar que não houve seleção de pacientes com uso exclusivo de TNP, sendo que, em sua maioria, receberam concomitantemente leite materno, fórmulas artificiais ou outro tipo de dieta enteral. Logo, a perda de peso corpóreo nos 6 primeiros dias de TNP pode estar relacionada a outras variáveis. A condição de menor aporte energético pela TNP, somada ao período de transição dos recém-nascidos (em média 5 dias de vida), justifica a perda de peso, que pode atingir até $15-20 \%$, reflexo da contração do volume extracelular ${ }^{16}$. Além disso, há uma perda considerável de água através da pele, trato respiratório, função renal imatura e espaço extracelular expandido ${ }^{17}$. Portanto, o fator intrínseco de perda de peso pós-natal deve ser considerado. No estudo de Guzmán et al. ${ }^{17}$, foram comparadas e detectadas as perdas de peso em $11,22 \%$ e 14,0\% para o grupo $\mathrm{A}(<1000 \mathrm{~g})$ e grupo B (1000-1250 g), respectivamente, e determinada a perda máxima de peso no período de 5,23 \pm 2,31 dias após o nascimento ${ }^{17}$.

Verifica-se que, quanto mais prematuro e menor o peso de nascimento do paciente (grupo B), maior é a diferença entre os volumes prescritos e preparados $(p=0,0003)$, pois o volume acrescido no preparo mantém-se constante (55 $\mathrm{mL}$ ), independente do volume necessário ao paciente. Como resultado, mantém-se as diferenças de volume prescrito e infundido $(p=0,0732)$ e volume preparado e infundido $(p=0,0003)$ devido à padronização de acréscimo no preparo. Estas três variáveis são estatisticamente diferentes entre o grupo A e o grupo B, como visualizado na Tabela 4 .

Os estudos relacionados às análises econômicas de custos hospitalares com terapia nutricional são escassos e contemplam pacientes adultos. Entretanto, identifica-se que os principais custos em saúde ocorrem devido às internações hospitalares ( $60 \%$ dos custos) e às terapias ( $10 \%$ dos custos), sendo que $57,7 \%$ dos custos com internações estão relacionados a materiais e medicamentos, incluindo os insumos para terapia nutricional ${ }^{16}$.

Nas contas hospitalares, verifica-se, também, que aproximadamente $35,44 \%$ do valor total dos custos estão relacionados às terapias nutricionais, sendo $40,18 \%$ na modalidade parenteral ${ }^{16}$. Confirma-se a representatividade financeira das terapias nutricionais nas contas hospitalares, principalmente quando se observam os gastos importantes com a TNP na administração em pacientes neonatos. Analisando os volumes médios preparados $(166,2 \mathrm{~mL}$ ) obtém-se um custo médio por bolsa de TNP de R $\$ 850,94$. Quando comparado com os valores encontrados das perdas monetárias dos volumes desprezados (média de $R \$ 203,10$ ), verifica-se um percentual de $23,87 \%$ de perdas no decorrer do processo de preparo e administração.

Quanto às limitações do estudo, algumas condições foram observadas. Trata-se de um estudo observacional e uma amostra pequena, com pacientes apresentando estado crítico, porém portadores de doenças com morbidades diferentes. $O$ uso de TNP isolada ou associada a outras estratégias de terapia nutricional, aliado a um tempo reduzido de dias consecutivos de TNP dos participantes do estudo, prejudicou uma avaliação correta das perdas do ponto de vista nutricional.

Conjuntamente, o parâmetro financeiro utilizado para mensurar as perdas monetárias foi considerado o valor comercializado em empresa privada local, enquanto a coleta dos dados foi realizada em um hospital público, que presta o serviço de preparo da TNP, impossibilitando a apuração real dos custos e perdas no processo. Entretanto, em consulta ao Setor de Avaliação e Controladoria do hospital, foi possível uma estimativa de apuração dos custos médios das TNP (insumos e mão de obra direta) por meio do método de custeio variável. Foi apurado um valor de $R \$ 1,99$ (um real e noventa e nove centavos) por $\mathrm{mL}$ de solução preparada. Contudo, os custos fixos e custos relacionados a equipamentos e manutenção da estrutura predial, assim como a tecnologia e material usados no preparo e administração, não foram imputados.

A escassez de trabalhos relacionados à TNP em RNs prematuros, principalmente quanto à apuração da suplementação energética adequada, dificultou a discussão dos resultados em vários tópicos. 
Diante dos resultados obtidos, constatado o acréscimo excessivo do volume de $55 \mathrm{~mL}$ padronizado para todas as preparações, e consequentes quantitativos de volumes descartados devido a este protocolar excesso, o que determina conseguinte perdas monetárias quantificadas por este estudo, será recomendado aos setores envolvidos a reavaliação e readequação dos volumes de preparo da TNP, além da revisão do protocolo institucional padronizado. A gestão na área da saúde tem o foco centrado na assistência e na segurança dos pacientes, porém sem deixar de considerar custos desnecessários que podem comprometer a sua própria sustentabilidade. Assim, acredita-se que tal medida proporcionará contribuição econômica positiva para a Instituição.

Recomendam-se novos estudos de análise econômica para avaliar os custos relacionados ao preparo de TNP e respectivas perdas, comparados aos valores faturados pela Instituição.

\section{CONCLUSÃO}

Os volumes de TNP infundidos são menores que os prescritos, resultando em provável prejuízo do aporte calórico para os pacientes neonatos críticos. Do mesmo modo, perdas financeiras significativas são associadas ao descarte de nutrição parenteral preparada e não administrada.

\section{REFERÊNCIAS}

1. Calkins KL, Venick RS, Devaskar SU. Complications associated with parenteral nutrition in the neonate. Clin Perinatol. 2014;41(2):331-45.

2. Tavares de Araujo VM, Gomes PC, Caporossi C. Enteral nutrition in critical patients: should the administration be continuous or intermittent? Nutr Hosp. 2014;29(3):563-7.

3. Pietz J. Feeding and fasting in the neonatal intensive care unit. JPEN J Parenter Enteral Nutr. 2015;39(6):621.

4. Shiroma GM, Horie LM, Castro MG, Martins JR, Bittencourt AF, Logullo L, et al. Nutrition quality control in the prescription and administration of parenteral nutrition therapy for hospitalized patients. Nutr Clin Pract. 2015;30(3):406-13.

5. Brennan AM, Kiely ME, Fenton S, Murphy BP. Standardized parenteral nutrition for the transition phase in preterm infants: a bag that fits. Nutrients. 2018;10(2):170.

6. Cotogni P. Management of parenteral nutrition in critically ill patients. World J Crit Care Med. 2017;6(1):13-20.

7. Moya DA, Gaitám AP, Camacho DO, Márquez HA. Hospital malnutrition related to fasting and underfeeding: is it na ethical issue? Nutr Clin Pract. 2016;31(3):316-24.

8. Neves A, Pereira-Da-Silva L, Fernandez-Llimos F. Neonatal parenteral nutrition prescription practices in Portugal. An Pediatr (Barc). 2014;80(2):98-105.

9. Dock-Nascimento DB, Tavares VM, Aguilar-Nascimento JE. Evolution of nutritional therapy prescription in critically ill patients. Nutr Hosp. 2005;20(5):343-7.

10. Hamilton S, McAleer DM, Ariagno K, Barrett M, Stenquist N, Duggan CP, et al. A stepwise enteral nutrition algorithm for critically iII children helps achieve nutrient delivery goals. Pediatr Crit Care Med. 2014;15(7):583-9.

11. Pradelli L, Graf S, Pichard C, Berger MM. Supplemental parenteral nutrition in intensive care patients: a cost saving strategy. Clin Nutr. 2017;37(2):573-9.

12. Brasil. Ministério da Saúde. Secretaria de Vigilância Sanitária. Portaria MS/SNVS nº 272, de 08 de abril de 1998. Aprova o Regulamento Técnico para fixar os requisitos mínimos exigidos para a Terapia de Nutrição Parenteral. Diário Oficial da União da República Federativa do Brasil. Brasília: Ministério da Saúde; 1998.

13. WHO Guidelines Approved by the Guidelines Review Committee. Pregnancy, childbirth, postpartum and newborn care: a guide for essential practice. 3rd ed. Geneva: World Health Organization; 2015.

14. Bolisetty S, OsbornD, Sinn J,LuiK; Australasian NeonatalParenteral Nutrition Consensus Group. Standardized neonatal parenteral nutrition formulations: an Australasian group consensus 2012. BMC Pediatr. 2014; 14:48.

15. Pappoe TA, Wu SY, Pyati S. A randomized controlled trial comparing an aggressive and a conventional parenteral nutrition regimen in very low birth weight infants. J Neonatal Perinatal Med. 2009;2(3):149-56.

16. Hyeda A, Costa ÉSMD. Economic analysis of costs with enteral and parenteral nutritional therapy according to disease and outcome. Einstein (Sao Paulo). 2017;15(2):192-9.

17. Guzmán JM, Jaraba MP, De La Torre MJ, Ruiz-González MD, Huertas MD, Alvarez R, et al. Parenteral nutrition and immature neonates. Comparativestudy ofneonatesweighingunder 1000 and 1000-1250 g at birth. Early Hum Dev. 2001;65(Suppl):S133-44.

Local de realização do estudo: Hospital Universitário Júlio Muller/ Universidade Federal de Mato Grosso, Programa de Mestrado Profissional, Cuiabá, MT, Brasil.

Conflito de interesse: Os autores declaram não haver. 